\title{
A20 Attenuates Lipopolysaccharide-Induced Inflammation Through MAPK/ERK/JNK Pathway in LX-2 Cells
}

\author{
Xiaohan Wang ${ }^{1,{ }^{*}}$, Feng $\operatorname{Han}^{1}$, Yueyu Shen ${ }^{2}$, Yunqing Chen ${ }^{2}$ and Zizhong Ji ${ }^{1}$ \\ ${ }^{1}$ Department of Gastroenterology and Hepatology, Affiliated Hospital of Jiaxing University, Jiaxing, Zhejiang, China \\ ${ }^{2}$ Department of Infectious Diseases, Affiliated Hospital of Jiaxing University, Jiaxing, Zhejiang, China \\ "Corresponding author: Department of Gastroenterology and Hepatology, Affiliated Hospital of Jiaxing University, 1882 Central-South Road, Jiaxing, 314000, Zhejiang Province, \\ China.Email:853706741@qq.com
}

Received 2021 February 24; Revised 2021 May 07; Accepted 2021 May 15.

\begin{abstract}
Background: Hepatic stellate cells (HSCs) are liver-specific pericytes that transform into myofibroblasts, which are involved in pathological vascularization in liver fibrosis. We previously suggested that A20 overexpression suppresses lipopolysaccharide(LPS)induced inflammation in HSC. We aimed to determine the mechanisms of the anti-inflammatory role of A20 in LX-2 cells.

Methods: LX-2 cells were transfected with A20-siRNA or control-siRNA and control adenovirus or A20-carrying adenovirus. Quantitative reverse transcription PCR (RT-qPCR) analysis was employed to quantify mRNA levels of $\alpha$-SMA, col-I, col-III, IL-6, TGF- $\beta$, and PDGF in A20-siRNA LX-2 cells stimulated with LPS. Multiple molecular indices of MAPK/ERK/JNK signal pathway were performed by using Western blotting.

Results: Relative to control, the fibrosis-related mRNA levels of $\alpha$-SMA, col-I, and col-III were increased in A20-siRNA LX-2 cells. Meanwhile, A20-siRNA cells significantly increased IL-6, TGF- $\beta$, and PDGF mRNA levels. Relative to controls, stimulating A20 overexpressing LX-2 cells with LPS for 5 and 30 minutes significantly reduced the levels of phosphorylated ERK and JNK, respectively. A20 knockdown in LX-2 cells promotes phosphorylated ERK and JNK levels with LPS for 30 minutes.

Conclusions: Our data indicate that A20 could be functional in HSCs through the MAPK/ERK/JNK signaling pathway, highlighting a potential novel therapeutic strategy against liver fibrosis.
\end{abstract}

Keywords: A20, Anti-inflammation, MAPK, ERK, JNK, LX-2 Cells

\section{Background}

Hepatic fibrosis in chronic liver disease is characterized by excessive collagen deposition and is a major public health concern $(1,2)$. During liver fibrogenesis, activation of hepatic stellate cells (HSCs) initiates processes leading to liver injury (3). Hepatic stellate cells are liver-specific pericytes that transform into myofibroblasts, which are involved in pathological vascularization in liver fibrosis (4). Previous studies have shown that hepatic inflammation is tightly associated with fibrosis (5).

Quiescent HSCs are vitamin A-storing cells located in the perisinusoidal space near the sinusoid endothelial cells (SEC) and hepatocytes (6). Hepatic stellate cells can become activated due to chemokines and cytokines released during liver injury. Activating signals include platelet-derived growth factors (PDGF), transforming growth factor- $\beta$ (TGF- $\beta$ ), and various cytokines, such as interleukin-6 (IL-6) (7). The continuous activation of HSCs can secret extracellular matrix (ECM) such as $\alpha$-smooth muscle actin ( $\alpha$-SMA), collagens, and inflammatory mediators, which contribute to hepatic inflammation and fibrosis (8). Thus, it is important to suppress hepatic inflammation in the initial stages of chronic liver diseases (7). Blocking or retarding the excessive inflammatory process could be a major therapeutic target for preventing hepatic fibrosis.

Zinc finger protein A20 has been found to negatively regulate inflammation with crucial physiological functions that protect the liver $(9,10)$. Lipopolysaccharide (LPS) can induce severe inflammation and enhance fibrosis in liver damage models (11). Treating human HSCs with LPS dramatically activates the inflammatory signaling pathway and aggravates the accumulation of proinflammatory chemokines (6). A20 is an intracellular ubiquitin-editing enzyme that has been shown as a crucial hepatoprotective factor to prevent chronic liver inflammation (12). A20 
plays an important role in liver protection through limiting inflammation following nonalcoholic fatty liver disease (NAFLD), chronic liver allograft dysfunction, hepatic ischemia-reperfusion injury, and acute liver failure (ALF) $(10,13-16)$. We previously suggested that A20 overexpression suppresses LPS-induced inflammation in HSC (17). However, the mechanisms through which A20 reduces inflammation in HSCs are unclear. Here, we evaluated the mechanisms of A20 anti-inflammatory effects in LX-2, a HSC cell line.

\section{Objectives}

In this study, we aimed to determine the mechanisms of the anti-inflammatory role of A20 in LX-2 cells via A20 overexpression and A20 knockdown.

\section{Methods}

\subsection{Ethical Approval Certificate}

The ethics committee of the hospital to Jiaxing University approved this study (No.2017-002). The study adhered to 1964 Helsinki Declaration guidelines.

\subsection{Cell Culture and Treatment}

LX-2 cells were obtained from ATCC and grown in DMEM (Gibco, Cat No.670087), containing 1\% pen/strep and $10 \% \mathrm{FBS}$ at $37^{\circ} \mathrm{C}$ in a humidified incubator, $5 \% \mathrm{CO}_{2}$. E. coli O055:B5 LPS (Sigma, Cat No.L6529) was used to stimulate the LX-2 cells at $0.1 \mu \mathrm{g} / \mathrm{mL}$, according to our previous research (17), for indicated durations before they were harvested and washed at least three times with PBS 1X for analysis.

\subsection{Design and Transfection of Adenovirus}

The control adenovirus and A20 overexpression adenovirus (Ad-A20) were designed by Obio Technology (shanghai) Co. Ltd. Shanghai, China. The adenovirus was produced and tittered as described (18). The two adenovirus preparations were transfected into LX-2 cells for $24 \mathrm{~h}$. The media were replaced with fresh media, and cells grown to $80-90 \%$ confluence. They were then rinsed with PBS $1 \mathrm{X}$ and cultured with serum-free DMEM/F12 before stimulation with LPS for indicated durations. Cells were then harvested and cryopreserved for later use.

\subsection{Design and Transfection of siRNA}

A20-siRNA (forward 5'-UAAGAUUGUCCCAUUCAUCTT-3', reverse 5'-GAUGAAUGGGACAAUCUUATT-3') and control siRNA (forward 5'-UUCUCCGAACGUGUCACGUTT-3', reverse 5'-ACGUGACACGUUCGGAGAATT -3') were designed by KeyGEN BioTechnology Co. Ltd. Nanjing, China. Lipofectamine 3000 (Invitrogen, CA, USA) was used to transfect control siRNA and A20-siRNA into LX-2 cells in 6-well cell culture plates. The concentration of control siRNA and A20-siRNA was $100 \mathrm{nM}$. The media were replaced with fresh media after $4-6 \mathrm{~h}$ of transfection, and cells were grown to $80-90 \%$ confluence. They were then rinsed with PBS 1X and cultured with serum-free DMEM/F12 before stimulation with LPS for indicated durations. Cells were then harvested and cryopreserved for later use.

\subsection{Western Blotting}

The cell samples were washed with PBS and digested with $1 \%$ Triton X-100 supplemented with protease inhibitors, $150 \mathrm{mM} \mathrm{NaCl}$, and 10\% glycerol for $30 \mathrm{~min}$ at $4^{\circ} \mathrm{C}$. Lysates were cleared by centrifugation at $4^{\circ} \mathrm{C}$ for $15 \mathrm{~min}$. The quantity of the extracted proteins was obtained using bicinchoninic acid (BCA) assay (Thermo Fisher Scientific, Cat No.23225). Next, $30 \mu$ g of protein samples was resolved on SDS-polyacrylamide gel and electrotransferred onto nitrocellulose membranes (Pall, Cat No.66485) before probing with anti A20 (1:1000), ERK (1:1000), phosphorylated ERK (1:400), JNK (1:1000), and phosphorylated JNK (1:1000) primary antibodies obtained from Cell Signaling Technology. The membranes were incubated with primary antibodies at $4^{\circ} \mathrm{C}$ overnight.

After washing in TBST three times, the samples were treated with horseradish peroxidase (HRP)-conjugated goat anti-rabbit or mouse IgG for 1 hour at room temperature. Blots were visualized by using enhanced chemiluminescence (ECL) kit (Millipore, MA, USA) and detected by the G:BOX chemiXR5 imager. The intensities of the protein bands were quantified by ImageJ software and calculated as a ratio of phosphorylated ERK and phosphorylated JNK to total ERK and JNK. Glyceraldehyde 3-phosphate dehydrogenase (GAPDH) was used as the loading control.

\subsection{RNA Extraction and RT-qPCR Analysis}

Cells were treated with Trizol to extract RNA, which was reverse-transcribed at $37^{\circ} \mathrm{C}$ for $15 \mathrm{~min}$ and at $85^{\circ} \mathrm{C}$ for $5 \mathrm{~s}$ in a $20 \mu \mathrm{L}$ reaction volume with PrimeScript ${ }^{\mathrm{TM}} \mathrm{RT}$ reagent Kit (Takara, Cat No.RR037A) following manufacturer instructions. Primers are presented in Table 1. For all the qRT-PCR 
experiments, primer efficiency was verified to be greater than 95\%. Quantitative reverse transcription PCR(RT-qPCR) assay was performed on a StepOnePlus ${ }^{\mathrm{TM}}$ Real-Time PCR system at $95^{\circ} \mathrm{C}$ for $5 \mathrm{~min}$, followed by 40 cycles at $95^{\circ} \mathrm{C}$ for $15 \mathrm{~s}$ and at $60^{\circ} \mathrm{C}$ for 20 s in a $20-\mu$ L reaction volume, as described in the manufacturer's guidelines on a StepOnePlus ${ }^{\mathrm{TM}}$ RealTime PCR System by Applied Biosystems (Applied Biosystems Inc., California, USA). Relative mRNA levels for the target genes were obtained by the $2(-\Delta \Delta \mathrm{Ct})$ method with GAPDH serving as the house-keeping gene.

\begin{tabular}{|c|c|}
\hline Primer & Sequence \\
\hline \multicolumn{2}{|l|}{$\alpha$-SMA } \\
\hline Forward & 5'-ACTGCCTTGGTGTGTGACAA-3' \\
\hline Reverse & 5'- CACCATCACCCCCTGATGTC -3' \\
\hline \multicolumn{2}{|l|}{ Col-I } \\
\hline Forward & 5'- GCTCGTGGAAATGATGGTGC -3' \\
\hline Reverse & 5'-ACCCTGGGGACCTTCAGAG -3' \\
\hline \multicolumn{2}{|l|}{ Col-III } \\
\hline Forward & 5'- TGCCCTACTGGTCCTCAGAACT -3' \\
\hline Reverse & 5'- CCTGCGAGTCCTCCTACTGCTA-3' \\
\hline \multicolumn{2}{|l|}{ TGF- $\beta$} \\
\hline Forward & 5'-AGGACCTCGGCTGGAAGTGGAT -3' \\
\hline Reverse & 5'-AGGACCTTGCTGTACTGCGTGT -3' \\
\hline \multicolumn{2}{|l|}{ IL-6 } \\
\hline Forward & 5'- CCTTCGGTCCAGTTGCCTTCTC -3' \\
\hline Reverse & 5'-AGAGGTGAGTGGCTGTCTGTGT -3' \\
\hline \multicolumn{2}{|l|}{ PDGF } \\
\hline Forward & 5'- GCTTGGCTCGTGGAAGAAGGAG -3' \\
\hline Reverse & 5'-TTGGCGTTGGTGCGGTCTATG -3' \\
\hline \multicolumn{2}{|l|}{ GAPDH } \\
\hline Forward & 5'- CAAATTCCATGGCACCGTCA-3' \\
\hline Reverse & 5'-AGCATCGCCCCACTTGATTT -3' \\
\hline
\end{tabular}

\subsection{Statistical Analysis}

All the data were analyzed using Graphpad Prism 5 (Graphpad, San Diego, CA) and are shown as mean \pm SD of three independent experiments. The mean values of different groups were compared using ANOVA or independent samples t-test. A P-value of less than 0.05 indicates statistical significance.

\section{Results}

4.1. A20 Suppresses LPS-induced Fibrosis-Related Molecules and Inflammatory Response in LX-2 Cells

Using immunohistochemistry, we previously observed elevated A20 levels in hepatic fibrosis patients (17). Here, we examined fibrosis-related molecules and inflammatory cytokine expression in control and A20-siRNA cells. Relative to controls, the fibrosis-related mRNA levels of $\alpha$-SMA, col-I, and col-III were increased in LX-2 cells transfected with A20-siRNA (Figure 1A). A20-siRNA cells significantly increased IL-6, TGF- $\beta$, and PDGF mRNA levels (Figure 1B), which activated HSCs (19), suggesting that A20 regulates these effectors of HSCs activation.

\subsection{MAPK/ERK/JNK Pathway Levels Are Elevated in LX-2 Cells in Response to LPS}

We previously found that LX-2 cells mount an inflammatory response and upregulate fibrosis indicators in response to LPS. Here, we focused on the MAPK/ERK/JNK signaling, which controls various processes in HSCs (20-22). Western blot analysis revealed elevated phosphorylated JNK and phosphorylated ERK levels in LX-2 cells relative to control (Figure 2). In order to explore whether A20 expression was involved in MAPK/ERK/JNK pathway, we overexpressed A20 via Ad-A20 and silenced A20 via A20-siRNA targeting A20 in LX-2 cells to analyze the changes.

\subsection{Function of A20 in MAPK/ERK/JNK Pathway}

We confirmed that inflammatory cytokines expression was elevated by silenced A20 in HSCs stimulated by LPS. However, the underlying mechanism is unknown. Thus, we focused on MAPK/ERK/JNK signaling pathways that influence liver fibrogenesis (23-26). Our analysis showed that relative to controls, A20 overexpression in LX-2 cells stimulated with LPS for different durations significantly reduced phosphorylated ERK levels after 5 minutes, and phosphorylated JNK levels were also lower at 30 minutes relative to controls (Figure $3 \mathrm{~A}-\mathrm{C}$ ). In the pre-experiment of A20 knockdown, we selected different durations and found that the band was most obvious at 30 minutes; thus, 30 minutes was chosen instead of the different durations according to A20 overexpression. A20 knockdown in LX-2 cells promotes phosphorylated JNK levels and phosphorylated ERK levels with LPS for 30 minutes (Figure 4A-C), suggesting that A20 modulates inflammation in HSCs via the MAPK/ERK/JNK pathway. 

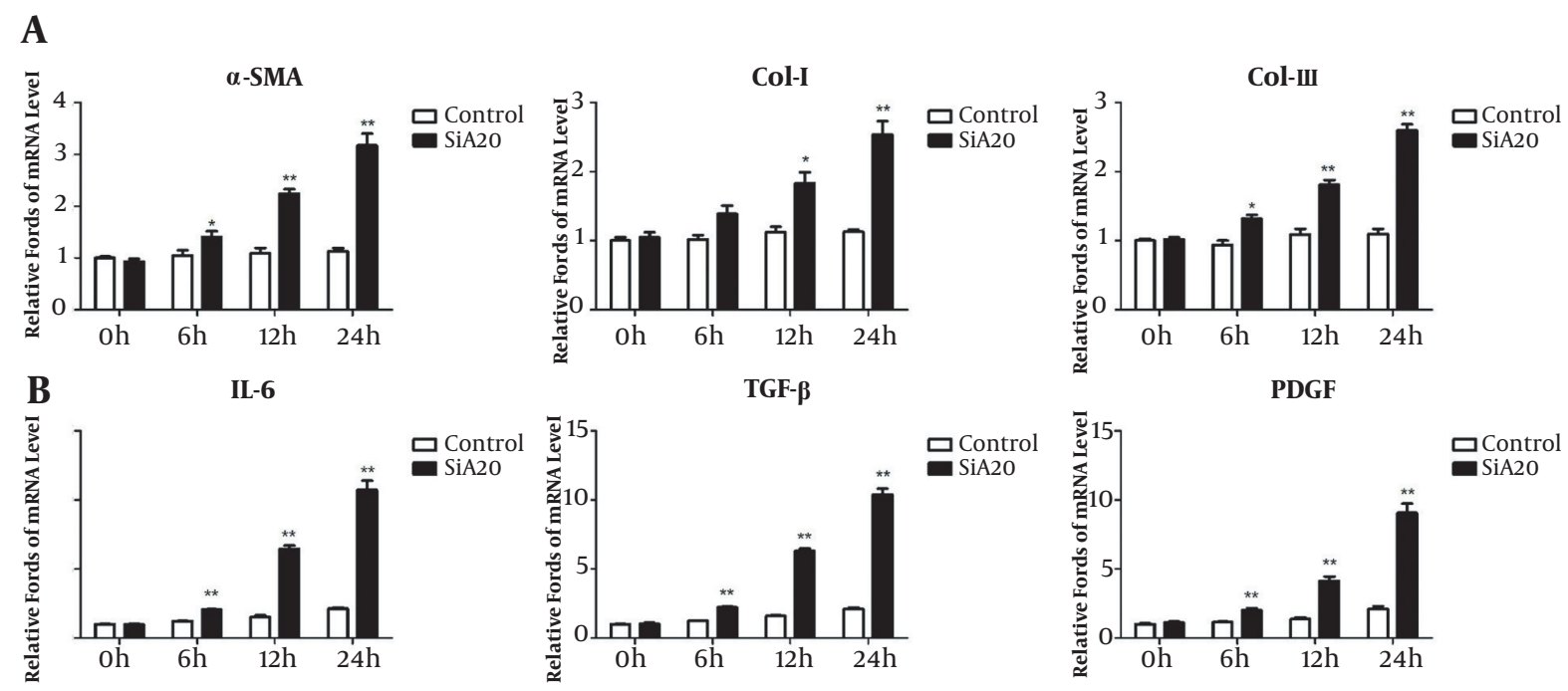

Figure 1. A20 knockdown (siRNA) promotes the mRNA expression of fibrotic markers and LPS-induced inflammatory response in LX-2 cells. A, The mRNA expression of $\alpha$-SMA, col-I, and col-III in LX-2 cells transfected with A20-siRNA and control exposed to various times of LPS. B, mRNA levels of IL-6, TGF- $\beta$, and PDGF in LX-2 cells transfected with A20-siRNA and control exposed to various times of LPS. GAPDH was used as the reference gene. ${ }^{*} \mathrm{P}<0.05$ and ${ }^{* *} \mathrm{P}<0.01$ relative to control groups.

\section{Discussion}

Here, we assessed the mechanism through which A20 mediates its anti-inflammation effects in LX-2 cells. The expression level of A20 is elevated in hepatic fibrosis tissue (17). Relative to controls, the fibrosis-related mRNA level of $\alpha$-SMA, col-I, and col-III were increased in A20-siRNA LX2 cells. Meanwhile, A20-siRNA cells significantly increased IL-6, TGF- $\beta$, and PDGF mRNA levels. Relative to controls, stimulating A20 overexpressing LX-2 cells with LPS for 5 and 30 minutes significantly reduced the levels of phosphorylated ERK and JNK, respectively. A20 knockdown in LX-2 cells promoted phosphorylated ERK and JNK levels with LPS for 30 minutes.

A20 influences various processes in the liver, including hepatic ischemia/reperfusion injury, liver protection, hepatocyte growth, hepatic inflammation, and apoptosis $(10,27,28)$. Previous studies showed that A20 promotes hepatocytes proliferation and suppresses apoptosis in ALF rats (29). Liver A20 expression enhanced BALB/c mouse survival after hepatectomy (28). In A20 knockout mice, excessive liver inflammation and necrosis led to compensatory physiologic A20 upregulation in hepatocytes (30). Additionally, A20 confers proliferative advantage in hepatocytes and is a potential therapeutic target against liver injury after ischemia reperfusion (13). Thus, A20 has proproliferative, anti-inflammatory, and antiapoptotic effects in hepatocytes (27). We previously found that A20 may pro- tect against liver fibrosis by downregulating inflammatory mediators in HSCs (17). A better understanding of A20's role in HSCs may uncover novel therapeutic approaches for reducing liver fibrosis.

Hepatic stellate cells play a central role in the development of liver fibrosis via promoting ECM formation in hepatic tissues. Chronic liver inflammation alters HSCs from a quiescent state to activated state, myofibroblasts, and fibroblasts, via stimulation by cytokines, including IL-6, PDGF, and TGF- $\beta$ (31, 32). IL-6 is a well-known proinflammatory cytokine, which can lead to liver injury and the occurrence of fibrosis (33). In the development of liver fibrosis, PDGF acts as a potent mitogen or activator of HSCs (34). TGF- $\beta$ is one of the impotent growth factors associated with fibrosis progression in the liver (35). These cytokines induce the activation and proliferations of HSCs, which consequently result in liver fibrosis or cirrhosis (3135). In our research, A20-siRNA cells significantly increased IL-6, TGF- $\beta$, and PDGF mRNA levels. Therefore, it is both interesting and important to know how A20 is linked to the above proinflammatory and profibrotic cytokines.

Previous studies (36) have shown that MAPK/ERK/JNK signaling controls various processes in HSCs, including cell growth, differentiation, survival, and apoptosis. ERK signaling has been shown to enhance liver fibrogenesis (23). A20 has been reported to downregulate TNF $\alpha$ induced chemokine secretion in human colorectal can- 
A

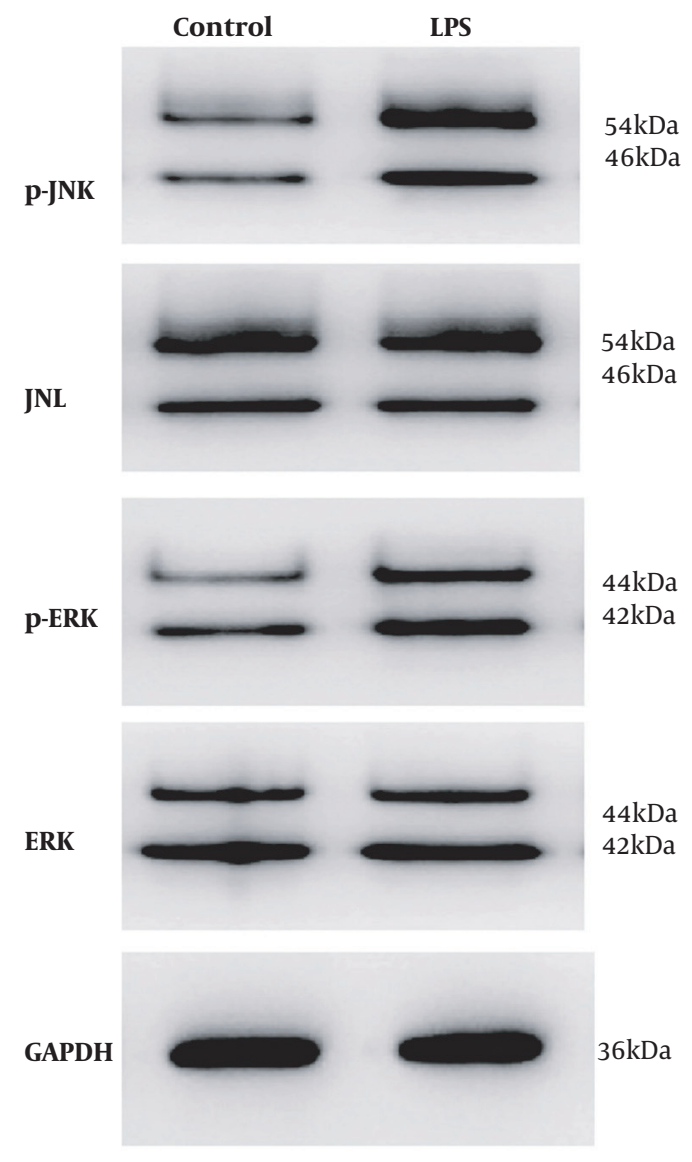

B
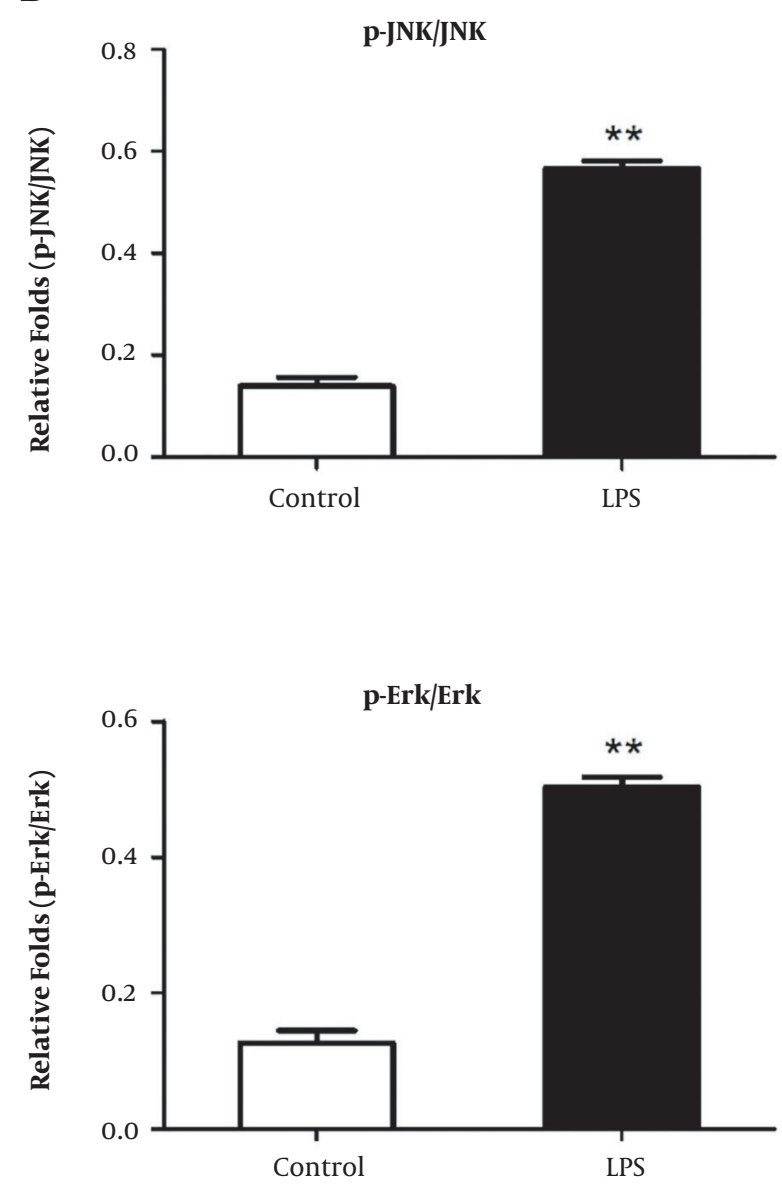

Figure 2. MAPK/ERK/JNK pathway levels are elevated in LX-2 cells. A, Western blot analysis of MAPK/ERK/JNK levels in LX-2 cells in response to LPS for 30 min. GAPDH served as the gene for loading control. B, Gray analysis of relative fold changes of protein phosphorylated JNK (p-JNK)/JNK and phosphorylated ERK (p-ERK)/ERK. P-JNK, JNK, p-ERK, and ERK represented as the sum of two bands. ${ }^{*} \mathrm{P}<0.05$ relative to control groups.

cer cells by suppressing ERK signaling (37). Moreover, inhibiting ERK and JNK phosphorylation in the MAPK pathway suppresses liver fibrosis (38). Some studies have found that A20 overexpression suppresses ERK and JNK activation, which are important for chemokine production $(37,39,40)$. These findings suggest that A20 negatively modulates chemokine production by suppressing MAPK/ERK/JNK signaling, and A20 has potential as a therapeutic target against liver fibrosis. However, the possibility that A20 may become physiologically upregulated in HSCs in response to inflammation during liver fibrosis needs further investigation.

A20 protects HSCs against inflammation by inhibiting MAPK signaling, highlighting this pathway as a potential target for liver fibrosis treatment.

\section{Acknowledgments}

We acknowledge the Zhejiang medical and health science and technology program (NO.2018KY798), Excellent Youth Foundation of Affiliated Hospital of Jiaxing University (NO.2018-GG-10), and Jiaxing Key Discipline (NO.2019zc-08) for funding this study.

\section{Footnotes}

Authors' Contribution: Study concept and design: Wang Xiaohan; Analysis and interpretation of data: Wang Xiaohan, Yunqing Chen, and Yueyu Shen; Drafting of the manuscript: Wang Xiaohan and Yueyu Shen; Critical revision of the manuscript for important intellectual content: 
A

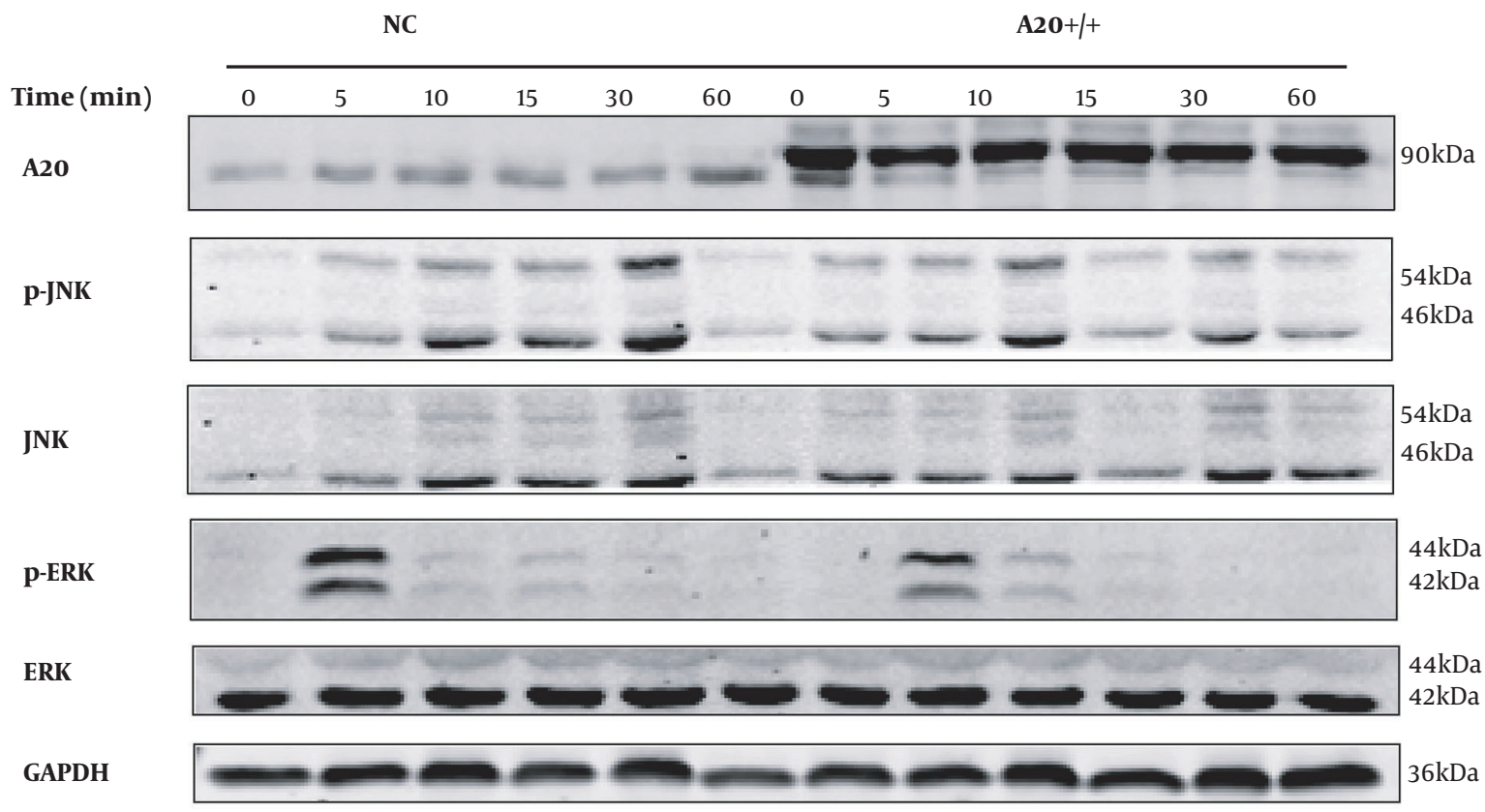

B

C
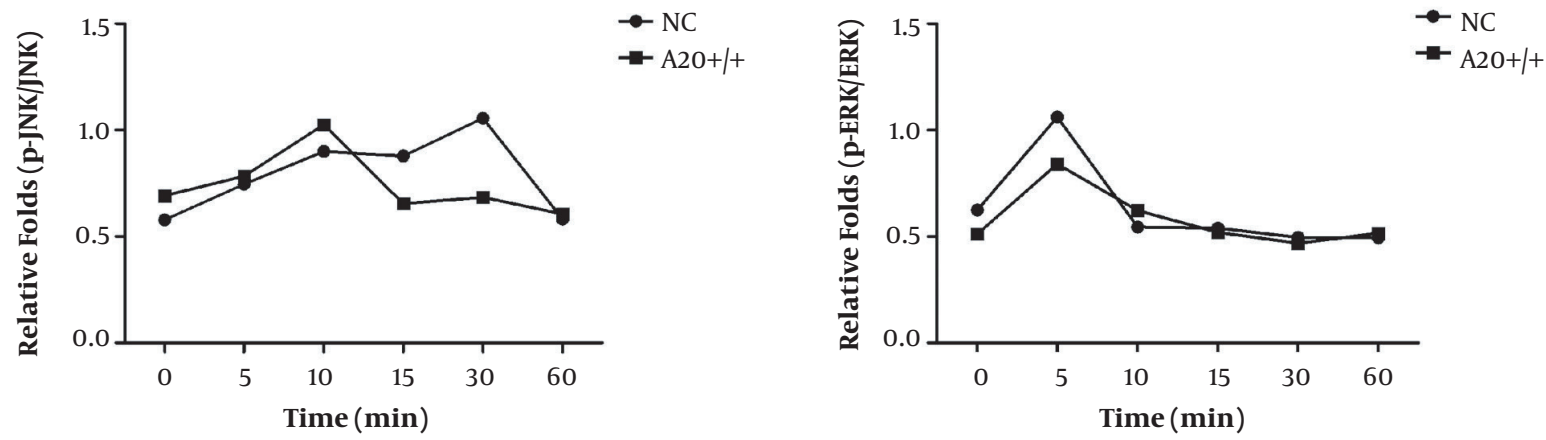

Figure 3. Function of A20 overexpression in MAPK/ERK/JNK pathway. A, Protein levels of phosphorylated MAPK/ERK/JNK in A20 overexpressing LX-2 cells exposed to 0.1 $\mu$ g/mL LPS for indicated durations. GAPDH served as the loading control. B, Graph shows relative fold changes of p-JNK/JNK. C, Graph shows relative fold changes of p-ERK/ERK. P-JNK, JNK, p-ERK, and ERK represented as the sum of two bands.

Wang Xiaohan; Statistical analysis: Wang Xiaohan, Feng Han, and Zizhong Ji.

Conflict of Interests: The authors declare no conflicts of interest.

Ethical Approval: The study was conducted with the approval of the ethics committee of the hospital affiliated to Jiaxing University (No.2017-002) and adhered to the 1964
Helsinki Declaration guidelines.

Funding/Support: We acknowledge the Zhejiang medical and health science and technology program (NO.2018KY798), Excellent Youth Foundation of Affiliated Hospital of Jiaxing University (NO.2018-GG-10), and Jiaxing Key Discipline (NO.2019-zc-08) for funding this study. 
A

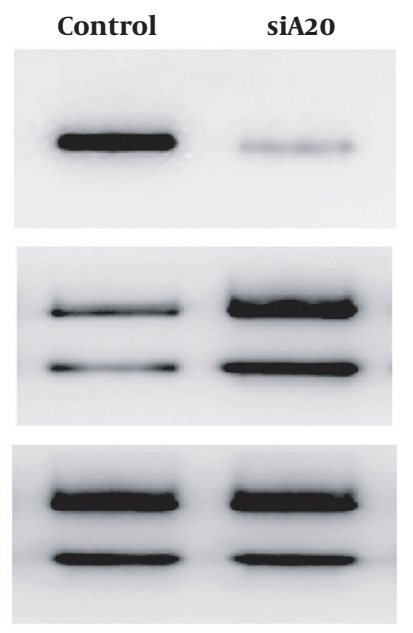

JNL

p-JNL

JNL

p-ERK

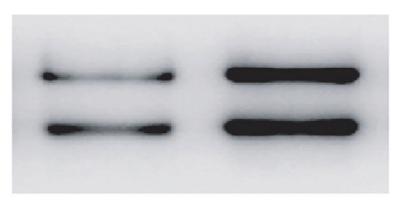

ERK

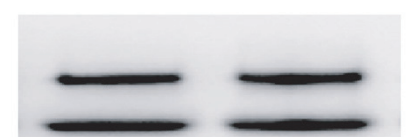

$54 \mathrm{kDa}$

$46 \mathrm{kDa}$

$54 \mathrm{kDa}$

$46 \mathrm{kDa}$

$44 \mathrm{kDa}$

$42 \mathrm{kDa}$

$36 \mathrm{kDa}$
B

p-JNK/JNK

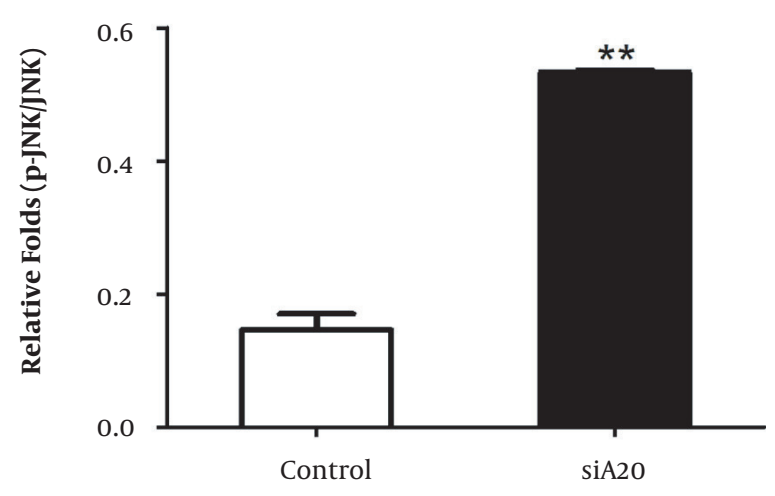

C

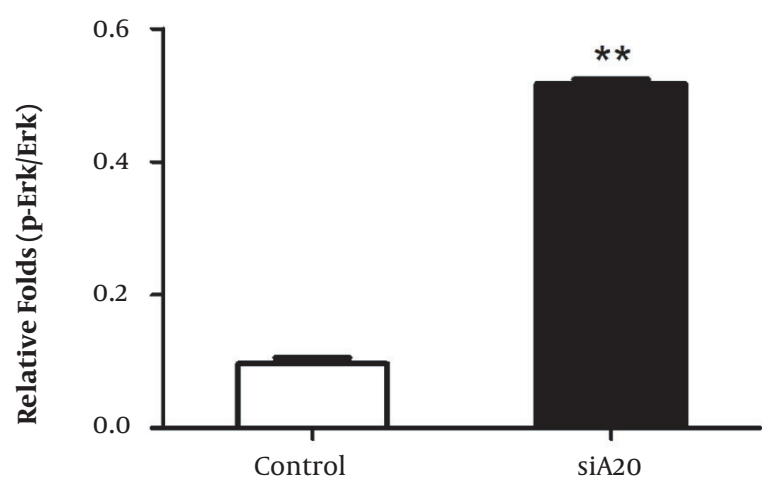

Figure 4. Function of silencing A20 in MAPK/ERK/JNK pathway. A, Protein levels of phosphorylated MAPK/ERK/JNK in LX-2 cells transfected with A20-siRNA exposed to $0.1 \mu \mathrm{g} / \mathrm{mL}$ LPS for 30 min. GAPDH served as the loading control. B, Graph shows relative fold changes of p-JNK/JNK. C, Graph shows relative fold changes of p-ERK/ERK. P-JNK, JNK, p-ERK, and ERK represented as the sum of two bands.

\section{References}

1. Tsuchida T, Friedman SL. Mechanisms of hepatic stellate cell activation. Nat Rev Gastroenterol Hepatol. 2017;14(7):397-411. doi: 10.1038/nrgastro.2017.38. [PubMed: 28487545].

2. Blachier M, Leleu H, Peck-Radosavljevic M, Valla DC, RoudotThoraval F. The burden of liver disease in Europe: A review of available epidemiological data. J Hepatol. 2013;58(3):593-608. doi: 10.1016/j.jhep.2012.12.005. [PubMed: 23419824].

3. Hernandez-Gea V, Friedman SL. Pathogenesis of liver fibrosis. Annu Rev Pathol. 2011;6:425-56. doi: 10.1146/annurev-pathol-011110-130246. [PubMed: 21073339].

4. Dou C, Liu Z, Tu K, Zhang H, Chen C, Yaqoob U, et al. P30o acetyltransferase mediates stiffness-induced activation of hepatic stellate cells into tumor-promoting myofibroblasts. Gastroenterology. 2018;154(8):2209-2221 e14. doi: 10.1053/j.gastro.2018.02.015. [PubMed: 29454793]. [PubMed Central: PMC6039101].
5. Hegde P, Weiss E, Paradis V, Wan J, Mabire M, Sukriti S, et al. Mucosalassociated invariant $\mathrm{T}$ cells are a profibrogenic immune cell population in the liver. Nat Commun. 2018;9(1):2146. doi: 10.1038/s41467-01804450-y. [PubMed: 29858567]. [PubMed Central: PMC5984626].

6. Wang C, Li Y, Li H, Zhang Y, Ying Z, Wang X, et al. Disruption of FGF signaling ameliorates inflammatory response in hepatic stellate cells. Front Cell Dev Biol. 2020;8:601. doi: 10.3389/fcell.2020.00601. [PubMed: 32793588]. [PubMed Central: PMC7387415].

7. Bak J, Je NK, Chung HY, Yokozawa T, Yoon S, Moon JO. Oligonol ameliorates $\mathrm{CCl}(4)$-induced liver injury in rats via the NF-kappa B and MAPK signaling pathways. Oxid Med Cell Longev. 2016;2016:3935841. doi: 10.1155/2016/3935841. [PubMed: 26798422]. [PubMed Central: PMC4699077].

8. Krenkel O, Hundertmark J, Ritz TP, Weiskirchen R, Tacke F. Single cell RNA sequencing identifies subsets of hepatic stellate cells and myofibroblasts in liver fibrosis. Cells. 2019;8(5). doi: 10.3390/cells8050503. [PubMed: 31137713]. [PubMed Central: PMC6562512]. 
9. Priem D, van Loo G, Bertrand MJM. A20 and cell deathdriven inflammation. Trends Immunol. 2020;41(5):421-35. doi: 10.1016/j.it.2020.03.001. [PubMed: 32241683].

10. Yi PS, Shu Y, Bi WX, Zheng XB, Feng WJ, He LY, et al. Emerging role of zinc finger protein A20 as a suppressor of hepatocellular carcinoma. J Cell Physiol. 2019;234(12):21479-84. doi: 10.1002/jcp.28877. [PubMed: 31134613].

11. Gaitantzi H, Karch J, Germann L, Cai C, Rausch V, Birgin E, et al. BMP-9 modulates the hepatic responses to LPS. Cells. 2020;9(3). doi: 10.3390/cells9030617. [PubMed: 32143367]. [PubMed Central: PMC7140468].

12. Catrysse L, Farhang Ghahremani M, Vereecke L, Youssef SA, Mc Guire C, Sze M, et al. A20 prevents chronic liver inflammation and cancer by protecting hepatocytes from death. Cell Death Dis. 2016;7(6). e2250. doi: 10.1038/cddis.2016.154. [PubMed: 27253414]. [PubMed Central: PMC5143384].

13. Yu J, Lee HS, Lee SM, Yu HC, Moon WS, Chung MJ, et al. Aggravation of post-ischemic liver injury by overexpression of A20, an NF-kappaB suppressor.J Hepatol. 2011;55(2):328-36. doi: 10.1016/j.jhep.2010.11.029. [PubMed: 21167234].

14. Sun J, Sun L, Zhang N, Lu X, Zhang H. A2O is up-regulated in primary mouse hepatocytes subjected to hypoxia and reperfusion. Cell Biochem Funct. 2012;30(8):683-6. doi: 10.1002/cbf.2850. [PubMed: 22736257].

15. Zhang P, Wang PX, Zhao LP, Zhang X, Ji YX, Zhang XJ, et al. The deubiquitinating enzyme TNFAIP3 mediates inactivation of hepatic ASK1 and ameliorates nonalcoholic steatohepatitis. Nat Med. 2018;24(1):84-94. doi: 10.1038/nm.4453. [PubMed: 29227477].

16. Yang J, Xu MQ, Yan LN, Chen XB, Liu J. Zinc finger protein A20 protects rats against chronic liver allograft dysfunction. World J Gastroenterol. 2012;18(27):3537-50. doi: 10.3748/wjg.v18.i27.3537. [PubMed: 22826618]. [PubMed Central: PMC3400855].

17. Wang X, Ai L, Xu Q, Wu C, Chen Z, Su D, et al. A20 attenuates liver fibrosis in NAFLD and inhibits inflammation responses. Inflammation. 2017;40(3):840-8. doi: 10.1007/s10753-017-0528-2. [PubMed: 28251449].

18. He TC, Zhou S, da Costa LT, Yu J, Kinzler KW, Vogelstein B. A simplified system for generating recombinant adenoviruses. Proc Natl Acad Sci U S A. 1998;95(5):2509-14. doi: 10.1073/pnas.95.5.2509. [PubMed: 9482916]. [PubMed Central: PMC19394].

19. Tu CT, Yao QY, Xu BL, Wang JY, Zhou CH, Zhang SC. Protective effects of curcumin against hepatic fibrosis induced by carbon tetrachloride: modulation of high-mobility group box 1, Toll-like receptor 4 and 2 expression. Food Chem Toxicol. 2012;50(9):3343-51. doi: 10.1016/j.fct.2012.05.050. [PubMed: 22683883].

20. Huang H, Wang K, Liu Q, Ji F, Zhou H, Fang S, et al. The active constituent from gynostemma pentaphyllum prevents liver fibrosis through regulation of the TGF-beta1/NDRG2/MAPK Axis. Front Genet. 2020;11:594824. doi: 10.3389/fgene.2020.594824. [PubMed: 33329740]. [PubMed Central: PMC7672159].

21. Zhang J, Jiang N, Ping J, Xu L. TGFbeta1induced autophagy activates hepatic stellate cells via the ERK and JNK signaling pathways. Int $J$ Mol Med. 2021;47(1):256-66. doi: 10.3892/ijmm.2020.4778. [PubMed: 33236148]. [PubMed Central: PMC7723502].

22. Pan LX, Li LY, Zhou H, Cheng SQ, Liu YM, Lian PP, et al. TMEM100 mediates inflammatory cytokines secretion in hepatic stellate cells and its mechanism research. Toxicol Lett. 2019;317:82-91. doi: 10.1016/j.toxlet.2018.12.010. [PubMed:30639579].

23. Foglia B, Cannito S, Bocca C, Parola M, Novo E. ERK pathway in activated, myofibroblast-like, hepatic stellate cells: A critical signaling crossroad sustaining liver fibrosis. Int J Mol Sci. 2019;20(11). doi: 10.3390/ijms20112700. [PubMed: 31159366]. [PubMed Central:
PMC6600376].

24. Wang L, Yang G, Yuan L, Yang Y, Zhao H, Ho CT, et al. Green tea catechins effectively altered hepatic fibrogenesis in rats by inhibiting ERK and Smad1/2 phosphorylation. J Agric Food Chem. 2019;67(19):5437-45. doi: 10.1021/acs.jafc.8b05179. [PubMed: 30424599].

25. Zhao G, Hatting M, Nevzorova YA, Peng J, Hu W, Boekschoten MV, et al. Jnk1 in murine hepatic stellate cells is a crucial mediator of liver fibrogenesis. Gut. 2014;63(7):1159-72. doi: 10.1136/gutjnl-2013-305507. [PubMed: 24037431].

26. Kluwe J, Pradere JP, Gwak GY, Mencin A, De Minicis S, Osterreicher $\mathrm{CH}$, et al. Modulation of hepatic fibrosis by c-Jun-Nterminal kinase inhibition. Gastroenterology. 2010;138(1):347-59. doi: 10.1053/j.gastro.2009.09.015. [PubMed: 19782079]. [PubMed Central: PMC2988578].

27. Ramsey HE, Da Silva CG, Longo CR, Csizmadia E, Studer P, Patel VI, et al. A20 protects mice from lethal liver ischemia/reperfusion injury by increasing peroxisome proliferator-activated receptor-alpha expression. Liver Transpl. 2009;15(11):1613-21. doi: 10.1002/lt.21879. [PubMed: 19877201]. [PubMed Central: PMC2976064].

28. Longo CR, Patel VI, Shrikhande GV, Scali ST, Csizmadia E, Daniel S, et al. A20 protects mice from lethal radical hepatectomy by promoting hepatocyte proliferation via a p21wafi-dependent mechanism. Hepatology. 2005;42(1):156-64. doi: 10.1002/hep.20741. [PubMed: 15962316].

29. Li KZ, Liao ZY, Li YX, Ming ZY, Zhong JH, Wu GB, et al. A20 rescues hepatocytes from apoptosis through the NF-kappaB signaling pathway in rats with acute liver failure. Biosci Rep. 2019;39(1). doi: 10.1042/BSR20180316. [PubMed: 30446523]. [PubMed Central: PMC6328859].

30. Studer P, da Silva CG, Revuelta Cervantes JM, Mele A, Csizmadia E, Siracuse JJ, et al. Significant lethality following liver resection in A20 heterozygous knockout mice uncovers a key role for A20 in liver regeneration. Cell Death Differ. 2015;22(12):2068-77. doi: 10.1038/cdd.2015.52. [PubMed: 25976305]. [PubMed Central: PMC4816110].

31. Lee SB, Kim HG, Kim HS, Lee JS, Im HJ, Kim WY, et al. Ethyl acetate fraction of amomum xanthioides exerts antihepatofibrotic actions via the regulation of fibrogenic cytokines in a dimethylnitrosamine-induced rat model. Evid Based Complement Alternat Med. 2016;2016:6014380. doi: 10.1155/2016/6014380. [PubMed: 27594891]. [PubMed Central: PMC4995331].

32. Zhang L, Wang X, Chen S, Wang S, Tu Z, Zhang G, et al. Medium-chain triglycerides attenuate liver injury in lipopolysaccharide-challenged pigs by inhibiting necroptotic and inflammatory signaling pathways. Int J Mol Sci. 2018;19(11). doi: 10.3390/ijms19113697. [PubMed: 30469452]. [PubMed Central: PMC6274951].

33. Qiao X, Wang L, Wang Y, Su X, Qiao Y, Fan Y, et al. Intermedin attenuates renal fibrosis by induction of heme oxygenase-1 in rats with unilateral ureteral obstruction. BMC Nephrol. 2017;18(1):232. doi: 10.1186/s12882 017-0659-6. [PubMed: 28697727]. [PubMed Central: PMC5505135].

34. Katsarou A, Moustakas ,I, Pyrina I, Lembessis P, Koutsilieris M, Chatzigeorgiou A. Metabolic inflammation as an instigator of fibrosis during non-alcoholic fatty liver disease. World J Gastroenterol. 2020;26(17):1993-2011. doi: 10.3748/wjg.v26.i17.1993. [PubMed: 32536770]. [PubMed Central: PMC7267690].

35. Khanjarsim V, Karimi J, Khodadadi I, Mohammadalipour A, Goodarzi MT, Solgi G, et al. Ameliorative effects of nilotinib on $\mathrm{CCl} 4$ induced liver fibrosis via attenuation of RAGE/HMGB1 gene expression and oxidative stress in rat. Chonnam Med J. 2017;53(2):118-26. doi: 10.4068/cmj.2017.53.2.118. [PubMed: 28584790]. [PubMed Central: PMC5457946].

36. Kuo LM, Chen PJ, Sung PJ, Chang YC, Ho CT, Wu YH, et al. The bioac- 
tive extract of Pinnigorgia sp. induces apoptosis of hepatic stellate cells via ROS-ERK/JNK-Caspase-3 signaling. Mar Drugs. 2018;16(1). doi: 10.3390/md16010019. [PubMed: 29315209]. [PubMed Central: PMC5793067].

37. Zhou X, An D, Liu X, Jiang M, Yuan C, Hu J. TNFalpha induces tolerant production of CXC chemokines in colorectal cancer HCT116 cells via A20 inhibition of ERK signaling. Int Immunopharmacol. 2018;54:296302. doi: 10.1016/j.intimp.2017.11.027. [PubMed: 29175508].

38. Takekoshi S, Kitatani K, Yamamoto Y. Roles of oxidized diacylglycerol for carbon tetrachloride-induced liver injury and fibrosis in mouse. Acta Histochem Cytochem. 2014;47(5):185-94. doi: 10.1267/ahc.14030.
[PubMed: 25861124]. [PubMed Central: PMC4387239].

39. Luo H, Liu Y, Li Q, Liao L, Sun R, Liu X, et al. A20 regulates IL-1induced tolerant production of CXC chemokines in human mesangial cells via inhibition of MAPK signaling. Sci Rep. 2015;5:18007. doi: 10.1038/srep18007. [PubMed: 26648169]. [PubMed Central: PMC4673611].

40. Song X, Yao Z, Yang J, Zhang Z, Deng Y, Li M, et al. HCV core protein binds to gC1qR to induce A20 expression and inhibit cytokine production through MAPKs and NF-kappaB signaling pathways. Oncotarget. 2016;7(23):33796-808. doi: 10.18632/oncotarget.9304. [PubMed: 27183919]. [PubMed Central: PMC5085119]. 